Results: A total of 151 children and young people (age range 2-17 years; Table 1) have been reported to the database from 12 countries; mostly Spain $(\mathrm{N}=30)$, France $(\mathrm{N}=29)$, Israel $(\mathrm{N}=29)$, and Czechia $(\mathrm{N}=25)$. Most patients had a diagnosis of juvenile idiopathic arthritis (JIA; N=92;61\%). Other diagnoses were autoinflammatory syndrome (including TRAPS, CAPS, FMF; 12\%), and systemic lupus erythematosus (4\%). There were $14(9 \%)$ hospitalisations and $1(0.7 \%)$ death reported due to COVID-19. The most commonly reported symptoms were fever (46\%), cough $(34 \%)$, anosmia $(19 \%)$, and headache (19\%). Only 19 (13\%) patients reported glucocorticoid use. DMARD therapy was used by 104 (69\%) patients; 67 (44\%) were on csDMARDs (methotrexate [N=54], antimalarials [N=7]), $45(30 \%)$ on anti-TNF, 9 (6\%) on IL-6 inhibitors, and 7 (5\%) on IL-1 inhibitors. Among the 145 patients with hospitalisation data, patients on any DMARD therapy (cs/b/tsDMARDs) had similar odds for hospitalisation compared with those not on therapy, adjusted for age (odds ratio $0.7 ; 95 \% \mathrm{Cl} 0.2,2.4)$.

\begin{tabular}{|c|c|c|}
\hline & & All Patients \\
\hline $\mathbf{N}$ & & 151 \\
\hline \multirow{3}{*}{ Gender } & Female & $94(62 \%)$ \\
\hline & Male & $56(37 \%)$ \\
\hline & Unknown & $1(<1 \%)$ \\
\hline \multirow[t]{2}{*}{ Age, years } & Median (IQR) & $12(8,15)$ \\
\hline & Range & 2 to 17 \\
\hline \multirow[t]{7}{*}{ Top Rheumatology Diagnoses } & Juvenile Idiopathic Arthritis (JIA) & $92(61 \%)$ \\
\hline & Polyarthritis & $50(33 \%)$ \\
\hline & Oligoarthritis & $31(21 \%)$ \\
\hline & Systemic & $11(7 \%)$ \\
\hline & Autoinflammatory syndrome (e.g. & $18(12 \%)$ \\
\hline & TRAPS, CAPS, FMF) & $6(4 \%)$ \\
\hline & Systemic Lupus Erythematosus & \\
\hline \multirow{4}{*}{ Comorbidities } & None stated & $112(74 \%)$ \\
\hline & Obesity & $9(6 \%)$ \\
\hline & Ocular inflammation & $9(6 \%)$ \\
\hline & Asthma & $3(2 \%)$ \\
\hline \multirow{3}{*}{ Required Hospitalisation } & Yes & $14(9 \%)$ \\
\hline & No & $131(87 \%)$ \\
\hline & Missing & $6(4 \%)$ \\
\hline \multirow{5}{*}{ Top 5 Symptoms Reported } & Fever & $69(46 \%)$ \\
\hline & Cough & $51(34 \%)$ \\
\hline & Anosmia & $28(19 \%)$ \\
\hline & Headache & $28(19 \%)$ \\
\hline & Fatigue & $23(15 \%)$ \\
\hline Deaths due to COVID-19 & Yes & $1(<1 \%)$ \\
\hline \multirow{10}{*}{ Treatment at onset of COVID-19 infection } & Glucocorticoids & $19(13 \%)$ \\
\hline & csDMARDs & $67(44 \%)$ \\
\hline & Methotrexate & $54(36 \%)$ \\
\hline & Antimalarials & $7(5 \%)$ \\
\hline & Mycophenolate & $5(3 \%)$ \\
\hline & bDMARDs & $64(42 \%)$ \\
\hline & Anti-TNF & $45(30 \%)$ \\
\hline & IL-6 & $9(6 \%)$ \\
\hline & $I L-1$ & $8(5 \%)$ \\
\hline & Any DMARD & $104(69 \%)$ \\
\hline
\end{tabular}

Conclusion: These initial data on outcomes of COVID-19 in paediatric RMDs are very reassuring, with less than 1 in 10 patients reporting hospitalisation. Due to the database design and inherent reporting bias, this is likely an overestimate, suggesting that overall outcomes among this population appear to be generally good, with mild infection. Increasing case reports to the database will allow further exploration of drug- and disease-specific outcomes.

Disclosure of Interests: None declared.

DOI: 10.1136/annrheumdis-2021-eular.1243

\section{POS1184 AUTOIMMUNE SYSTEMIC DISEASES AND COVID-19 INFECTION}

S. Monov ${ }^{1}$, R. Shumnalieva ${ }^{2}$, D. Monova ${ }^{3} .{ }^{1}$ Medical University - Sofia, Departmant of Rheumatology, Sofia, Bulgaria; ${ }^{2}$ Medical University - Sofia, Department of Rheumatology, Sofia, Bulgaria; ${ }^{3}$ Medical Institute, Medical University - Sofia, Department of Internal Medicine, Sofia, Bulgaria

Background: Covid-19 infection poses a serious challenge for immune-compromised patients. This is likely due to a combination of immune dysfunction, immunosuppressive therapy and excess co-morbidities. Little is known about the impact of Coronavirus disease 2019 (COVID-19) in patients with inflammatory autoimmune systemic diseases.

Objectives: The aim of this study is to describe clinical characteristics of patients with autoimmune systemic diseases and COVID-19, and to identify baseline variables associated with a severe infection requiring hospitalization.

Methods: A telephone survey investigating the impact of COVID-19 on patients with systemic lupus erythematosus (SLE), systemic sclerosis (SSc), inflammatory arthritis (rheumatoid arthritis, psoriatic arthritis, ankylosing spondylitis), idiopathic inflammatory myopathies (IIM), ANCA-associated vasculitis (AAV) was administered. Data extraction included diagnosis, disease activity status, demographics, disease duration, occupational exposure, adherence to social distancing advise, therapy, comorbidities, and laboratory tests. Covid-19 was classified as definite diagnosis of Covid-19 disease: presence of symptomatic Covid-19 infection, confirmed by a nasopharyngeal SARS-CoV-2 polymerase chain reaction test. Comparisons between patients with or without hospitalization were performed.

Results: 512 patients (median age 53,4 \pm 14,3 years) with autoimmune systemic diseases (234 IA, 182 SLE, 42 SSc, 31 IIM, 23 AAV) were included in the study. 89 patients (58 woman, 31 men) developed at least one symptom (fever, asthenia, chills, cough, sore throat, dyspnea, ches pain, headaches, arthralgias, myalgias, odynophagia, diarrhea, conjunctivitis, hypo-, ageusia, hypo-, anosmia) of COVID-19 and were PCR test positive. Of patients with COVID - 19 infection 54 patients were treated with methylprednisolone, 36 - with methotrexate, 34 - with hydroxychloroquine, 26- with biologics, 10 - with azathioprine, 6 - with cyclophosphamide prior to their COVID-19 illness.

Conclusion: Covid-19 is more frequent in the subgroup of patients without therapy with modifying anti-rheumatic drugs, which might play some protective role against the most harmful manifestations of Covid-19. 21 patients required hospitalization - these were more frequently men, older and with comorbidities (cardio-respiratory illness, renal diseases, diabetes mellitus). Male sex, previous coronary and lung disease, serum creatinine level, proteinuria, glucocorticoids use $>5 \mathrm{mg} /$ day, were associated to hospitalization. Patients with inflammatory arthritis do not seen to be at higher risk for infection or a severe course of COVID-19.

\section{REFERENCES:}

[1] Monova, D., S. Monov. Mechanisms of kidney injury in patients with COVID19. Nephrology, dialysis and transplantation, 2020; 26 (4): 5-15.

[2] Monova, D., S. Monov. Kidney injures in COVID-19. Nephrology, dialysis and transplantation, 2020; 26 (4): 16-34.

Disclosure of Interests: Simeon Monov Speakers bureau: AMGEN, PFIZER, NOVARTIS, ABBVIE, ROCHE, ASTRA-ZENECA, Russka Shumnalieva: None declared, Daniela Monova: None declared.

DOI: 10.1136/annrheumdis-2021-eular.1254

\section{POS1185 IMPACT OF LOCKDOWN DURING COVID-19 PANDEMIC ON THE ONSET OF POST-TRAUMATIC STRESS DISORDER (PTSD) IN SYSTEMIC SCLEROSIS PATIENTS: A CASE-CONTROL STUDY}

D. M. Reza Beigi ${ }^{1}$, G. Pellegrino ${ }^{1}$, A. Lorello ${ }^{1}$, C. Angelelli ${ }^{1}$, K. Stefanantoni ${ }^{1}$ F. Conti ${ }^{1}$, V. Riccieri ${ }^{1}$. 'Sapienza University of Rome, Department of Internal Medicine, Anesthesiology and Cardiovascular Sciences, Rome, Italy

Background: Social distancing due to COVID-19 pandemic had a major impact on the mental health of general population, with a high prevalence of post-traumatic stress disorder (PTSD) related symptoms ${ }^{1,2}$. Psychological repercussions were notably found in people with chronic diseases, including systemic sclerosis (SSc) patients, where an increasing of anxiety symptoms, related also to low financial resources, emerged ${ }^{3}$.

Objectives: To evaluate the impact of COVID-19 lockdown on the onset of PTSD in patients with SSc, firstly during the total confinement period (March-April 2020) and then at the time of less restrictive government measures, following the RT index lowering (June-July 2020) ${ }^{4}$.

Methods: We carried out a case-control study on 57 SSc patients, according to the ACR/EULAR 2013 criteria, and on 57 healthy subjects as control group (HC), matched by sex and age. At T0 (March-April) and T1 (June-July) both populations received the "Impact of Event Scale Revised" questionnaire (IES-R) by e-mail, with a cut-off of $\geq 33$ defining probable diagnosis of $\mathrm{PTSD}^{5}$. A multivariate analysis of possible factors influencing IES-R score, such as age, number of cohabitating people and weekly outings count, was performed in SSc patients at both times of the survey.

Results: At T0 we found a significantly greater number of SSc patients with IES-R score $\geq 33$ compared to HC (26/45.6\% vs $13 / 22.8 \%$; median value [quartiles] 31 [19.5;42.5] vs 24 [15.5; 32]; p-value 0.046). At T1, we obtained data from $44 \mathrm{SSc}$ patients and $35 \mathrm{HC}$ but no significant difference was noticed ( $18 / 40.9 \%$ vs $8 / 23.5 \% ; 26$ [15.25; 38] vs 26.5 [20.75; 32.5]; $p>0.05)$. SSc patients also had significantly fewer weekly outings than HC, both at T0 ( $<<0.001)$ and T1 ( $<<0.001)$ (Table 1). The multivariate analysis performed at TO on SSc patients showed a significant association of IES-R $\geq 33$ score with age ( $p$ 0.025) and with a lower count of weekly outings ( $p$ $0.002)$. The latter data negatively correlated with an IES-R $\geq 33$ score in SSc patients ( $r-0.267, \mathrm{p} 0.004)$.

Conclusion: We found a significantly higher prevalence of PTSD in SSc patients compared to $\mathrm{HC}$ at the strictest lockdown time, turning into comparable when 